\title{
Les poisons de flèches utilisés par les Pygmées de la préfecture de la Lobaye (République Centrafricaine)
}

\author{
Olga Diane YONGO $^{1 *}$, Aristide-Kévin PONIBANDA ${ }^{1}$ et Bruno de FOUCAULT ${ }^{2}$ \\ ${ }^{I}$ Université de Bangui, Faculté des Sciences, Laboratoire de Biodiversité Végétale et Fongique, \\ BP 908, avenue des Martyrs, Bangui, République Centrafricaine. \\ ${ }^{2}$ Département de Botanique, Faculté de Pharmacie, BP 83, F-59006 Lille Cedex, France. \\ *Auteur correspondant ; E-mail : odiyongo@yahoo.fr ; Tél. (+ 236) 70978610
}

\section{RESUME}

Les détenteurs des savoirs traditionnels sont généralement des personnes âgées. Souvent, celles-ci meurent sans transmettre leurs connaissances aux jeunes de plus en plus désintéressés. Ainsi, avant que cela ne soit trop tard, nous nous sommes fixés pour objectif de rassembler les informations sur les poisons de flèches utilisés par les Pygmées de la préfecture de la Lobaye. Pour cela, nous avons conduit des enquêtes ethnobotaniques. Quinze personnes ont été interrogées, quatorze Pygmées Aka et un «villageois». Au total, nous avons recensé 25 espèces de plantes pour lesquelles 21 sont indiquées comme entrant dans la préparation des poisons de flèches dans cette région ; les quatre autres espèces sont utilisées pour la fabrication d'arcs, d'arbalètes et de sagaies. Treize des 25 espèces sont signalées dans la littérature comme étant utilisées comme poisons de flèches. Periploca nigrescens Afzel. (Apocynaceae) constitue l'élément principal de plusieurs poisons.

(C) 2014 International Formulae Group. All rights reserved.

Mots clés : Poisons de flèches, Pygmées, enquêtes ethnobotaniques, République Centrafricaine.

\section{INTRODUCTION}

L'étude des poisons de flèches a toujours contribué à de grands progrès dans bien de disciplines scientifiques telles que la pharmacologie, la toxicologie, la physiologie, l'anatomopathologie, l'électrophysiologie, etc. (Chevalier, 1951; Angenot, 1978 ; Jassaud, 1991 ; Ciccone, 2011). Outre ces importantes découvertes scientifiques, les pratiques anciennes de chasse présentent un caractère purement artistique. Il s'agit là d'un art à part entière : l'art d'empoisonner par le biais des projectiles confectionnés traditionnellement. Depuis la fabrication d'arcs, d'arbalètes, de lances ou de sagaie, jusqu'à l'empoisonnement des pointes de ces outils, il se dessine tout un ensemble de savoirs et savoir-faire traditionnels propres à un continent, à un pays et/ou à une région donnée mais également à des groupes d'individus d'un même groupe social. On notera éventuellement une certaine particularité morphologique et dimensionnelle de ces outils et l'utilisation des substances naturelles (animale, végétale, fongique et minérale) servant ou entrant dans la composition de ces poisons suivant les régions étudiées. Afin de contribuer à la sauvegarde des savoirs et connaissances traditionnels, nous nous sommes fixés pour objectif de rassembler les 
informations sur les savoir-faire anciens des Pygmées Aka et de faire l'inventaire des espèces végétales utilisées dans les pratiques traditionnelles de chasse et de pêche. Les Pygmées Aka sont considérés comme les premiers occupants de l'écosystème forestier centrafricain (Kalk, 1992). Ils vivaient jadis en harmonie avec la forêt, de la chasse, de la pêche et de la cueillette. Mais actuellement, ils ont tendance à se détourner d'une bonne partie de leurs pratiques traditionnelles. En effet, les tactiques actuelles de chasse et de pêche font intervenir de plus en plus d'armes à feu et des filets synthétiques au lieu de leurs propres outils (arcs, arbalètes, lances, sagaies, filets artisanaux, etc.). Il est pour nous très urgent de pouvoir compléter la littérature sur ces valeurs ancestrales faute de quoi elles pourront disparaître.

\section{MATERIEL ET METHODES}

L'étude s'est déroulée dans la préfecture de la Lobaye localisée dans le sudouest de Centrafrique. Elle s'étend sur environ $18511 \mathrm{~km}^{2}$ avec une population totale d'environ 214137 habitants. C'est dans la commune de Mbata $\left(18^{\circ} 17^{\prime}\right.$ E et $\left.3^{\circ} 40^{\prime} \mathrm{N}\right)$, plus précisément dans le village de Mokinda $\left(18^{\circ} 21^{\prime} \mathrm{E}\right.$ et $\left.3^{\circ} 40^{\prime} \mathrm{N}\right)$ et ses environs (Wèrèwèrè, Sakoungou, Sikilongo, Motomato, Moundjou ngonda) que nous avons effectué les travaux.

Le climat du milieu d'étude est de type guinéen forestier (Aubréville, 1948) caractérisé par une saison des pluies longue de neuf mois, de deux mois d'intersaison et un mois de saison sèche. La moyenne des précipitations est de l'ordre de $1490 \mathrm{~mm} / \mathrm{an}$. La température reste toujours élevée avec une moyenne annuelle de $26{ }^{\circ} \mathrm{C}$. La végétation est la forêt dense semi-décidue actuellement assez secondarisée par endroit. Le substrat géologique est constitué de grès quarzite. Les sols de la zone sont majoritairement de type ferralitique et de fertilité relativement faible (Boulvert, 1996).

\section{Méthodes Enquêtes ethnobotaniques}

Avant de procéder aux enquêtes dans chacun des villages, nous avons eu des entretiens avec les autorités de la place (chefs du quartier, notables...) afin de recueillir l'adhésion de la population, plus particulièrement des Pygmées qui sont considérés comme les autochtones de la localité. Nous avons demandé aux Pygmées de se rendre plus disponibles pour quelque temps car ils sont souvent retenus pour des travaux champêtres, la chasse, la cueillette des différentes espèces de Gnetum très prisées comme légumes. Après avoir gagné la confiance de la population, accompagnés d'un villageois, nous avons parcouru les campements des Pygmées pour identifier ceux qui connaissent les poisons de flèches utilisées pour la chasse ou la pêche. Les Pygmées sont en effet très réputés pour la connaissance des usages de nombreuses ressources forestières.

Pour les enquêtes proprement dites, à chaque personne identifiée, nous avons posé une série de questions concernant son savoir sur l'art d'empoisonnement et les substances utilisées; les réponses ont été systématiquement mentionnées ou cochées dans une fiche d'enquête préalablement établie. Les données des enquêtes ont été saisies et soumises à des analyses statistiques simples sur Excel.

\section{Récolte et détermination des échantillons botaniques}

Afin d'identifier scientifiquement les espèces, nous avons préparé un herbier. Nous avons suivi sur le terrain le praticien pour pouvoir mieux identifier sur pied les espèces végétales. Les échantillons de plantes ont été collectés au moins en deux exemplaires et déposés à l'herbier du Laboratoire de biodiversité végétale et fongique de l'Université de Bangui. Nous avons pris pour chaque spécimen des photos facilitant ainsi sa détermination. Nous avons déterminé le maximum d'échantillons au Laboratoire en consultant de nombreux ouvrages botaniques d'Afrique centrale et occidentale (Flore du 
Cameroun, 1963-1991 ; Flore du Congo, du Rwanda et du Burundi, 1948-1968; Flore du Gabon, 1961-1966 ; Flora of West Tropical Africa, 1958-1968). L'outil Internet nous a été également d'un grand support pour les vérifications. La nomenclature suivie pour les espèces est celle de Lebrun et Stork (1991, 1992, 1995, 1997). Pour les familles, nous nous sommes référés à l'APG III (Angiosperm Phylogeny Group) (2009).

\section{RESULTATS}

$\mathrm{Au}$ total, nous avons interrogé quinze personnes, tous des hommes, dans six villages. Nous avons eu à interviewer un villageois Ngbaka et quatorze Pygmées Aka, dont six Pygmées Aka « Ngbaka » (vivant en association avec les Ngbaka) et huit Pygmées Babènzè (Babènzèlè). Il est à noter que le nombre des enquêtés est ici assez restreint car très peu de Pygmées s'adonnent à l'empoisonnement des flèches pour la chasse ou la pêche de nos jours. Sur les quatorze Pygmées interviewés, treize disent avoir hérité de leurs parents ces pratiques et un, de ses amis ou connaissances. De toutes ces personnes interrogées, seulement six reconnaissent avoir partagé leurs connaissances à leurs proches sur les poisons de flèches. Selon les interviewés, actuellement, les jeunes Pygmées ne s'intéressent guère à ces valeurs ancestrales qu'ils considèrent comme révolues.

Les enquêtes réalisées dans cette région ont permis de collecter 25 espèces de plantes. $\mathrm{Au}$ total, 21 espèces ont été distinguées comme étant des ingrédients ou adjuvants des poisons de flèches. Les quatre autres espèces sont utilisées pour confectionner les arcs, les arbalètes, les sagaies. Ces espèces sont réparties dans vingt genres et quinze familles. Comme le montre la Figure 1, les familles les plus représentées en termes de nombre d'espèces sont les Apocynaceae $(21,7 \%)$, les Fabaceae $(8,7 \%)$, les Euphorbiaceae $(8,7 \%)$, les Loganiaceae $(8,7 \%)$ et les Sapotaceae $(8,7 \%)$. Et par rapport au nombre de citations, les
Apocynaceae sont fortement représentées $(32,4 \%)$, ensuite viennent les Dioscoreaceae (11,8\%), les Fabaceae (8,8\%), les Loganiaceae $(8,8 \%)$, les Euphorbiaceae $(5,9 \%)$ et les Sapotaceae $(5,9 \%)$. Pour la fabrication des poisons, les écorces, les feuilles et les tiges sont les plus utilisées (Figure 2). C'est surtout des lianes que les Pygmées tirent les poisons (Figure 3).

\section{Monographie sur les plantes utilisées comme poisons}

On présente ici les espèces, leurs caractéristiques et leurs propriétés.

Les espèces utilisées pour la préparation des poisons de flèches

*APOCYNACEAE

Nom scientifique : Periploca nigrescens

Afzel.

Nom vernaculaire : Mumbango (aka)

C'est une liane qui contient des hétérosides cardiotoniques. L'espèce constitue l'élément principal de plusieurs poisons utilisés par les Pygmées de la Lobaye.

Nom scientifique : Cascabela thevetia (L.) Lippold

Nom vernaculaire : Yoro ti béndé (sango)

Arbuste d'environ $8 \mathrm{~m}$ de haut; plante exotique (originaire d'Amérique tropicale), souvent cultivée comme plante ornementale. Toutes les parties de la plante sont signalées toxiques. Les Pygmées Aka de la Lobaye disent avoir utilisé le latex pour empoisonner les pointes des outils de chasse en les trempant dans l'exsudat recueilli. Les fruits sont utilisés par les villageois de la localité comme poison des rats.

Nom scientifique : Tabernaemontana crassa Benth.

Noms vernaculaires : Bõkõ (aka), Itopolopo (aka babènzè).

Tabernaemontana crassa est un petit arbre qui contient un grand nombre d'alcaloïdes indoliques avec d'intéressantes activités pharmacologiques. Les feuilles et le latex sont mélangés avec les feuilles et tiges de Periploca nigrescens, pilés et réduits en pâte qu'on applique sur les pointes des outils 
et qu'on laisse au moins deux jours avant l'utilisation pour la chasse. L'écorce des racines râpée, additionnée d'un peu d'eau servirait à traiter les maladies oculaires.

Nom scientifique : indéterminé

Nom vernaculaire : Ekombé (aka)

C'est une grande liane. Ses écorces râpées sont ajoutées à celles de Strychnos aculeata et $S$. camptoneura ; on fait bouillir l'ensemble avec les pointes des outils de chasse pour les empoisonner.

Nom scientifique : indéterminé

Nom vernaculaire : Mokéssa (aka)

C'est une grande liane. Les écorces du tronc sont bouillies avec celles de Pentaclethra macrophylla Benth., de litouma et de Manilkara sp. pendant quelques minutes jusqu'à obtenir un fluide sirupeux noirâtre qu'on applique sur les tranchants des outils de chasse. Le poison reste actif pendant un temps assez long.

*ARACEAE; Nom scientifique : Caladium bicolor (Ait.) Vent. Nom vernaculaire : Ndombi (aka)

C'est une plante herbacée naturalisée. La plante est signalée toxique par les Pygmées. Les autochtones de la Lobaye utilisent le tubercule découpé en morceaux bouillis avec les pointes des flèches pour les empoisonner.

\section{DIOSCOREACEAE}

Nom scientifique : Dioscorea sp.

Nom vernaculaire : Liblakènzè ou Libalkènzè (aka)

C'est une liane à tige cylindrique. C'est une igname toxique utilisée (le tubercule et les feuilles) par les Aka de la Lobaye pour la préparation des poisons de flèches.

\section{EUPHORBIACEAE}

Nom scientifique : Elaeophorbia grandifolia (Haw.) Croizat

Nom vernaculaire : Songo (ngbaka)

Arbuste à exsudat très abondant de latex blanc qui provoque une démangeaison au contact de la peau. C'est une plante toxique. Les Aka utilisent l'exsudat pour empoisonner les pointes des flèches et des sagaies en vue de la chasse. D'après eux, c'est un poison redoutable.

\section{FABACEAE}

Nom scientifique : Erythrophleum suaveolens (Guill. \& Perr.) Brenan

Nom vernaculaire : Gbanda (aka).

Arbre de grande taille, atteignant $40 \mathrm{~m}$ de haut, Erythrophleum suaveolens contient des alcaloïdes. L'écorce râpée ou pilée et réduite en pâte est appliquée, puis emballée autour des pointes des outils de chasse; ou les écorces morcelées sont bouillies avec les pointes des outils comme poison de flèche. On utilise aussi l'écorce râpée pour engourdir les poissons afin de les pêcher plus facilement.

\section{*GELSEMIACEAE.}

Nom scientifique : Mostuea hirsuta T. Anderson ex Benth. \& Hook. f.

Nom vernaculaire : Mõkõ-balé (ngbaka).

Mostuea hirsuta est un sous-arbrisseau qui contient des alcaloïdes indoliques. L'écorce des racines râpée est mélangée avec les feuilles de Periploca nigrescens pilées et réduites en pâte, le tout étant appliqué sur la lame des flèches. Et la poudre de racines (râpées) sert de breuvage mortel pour les animaux (y compris l'homme); il suffirait d'en verser dans une boisson (par exemple, vin de palme) une petite quantité pour ôter la vie d'une personne, si on ne réagit pas vite.

\section{LOGANIACEAE}

Nom scientifique : Strychnos aculeata Soler.

Nom.vernaculaire : Épombé (aka).

Grande liane grimpante, Strychnos aculeata contient des alcaloïdes indoliques. L'écorce du tronc, râpée ou morcelée, est ajoutée à celles de S. camptoneura et d'une autre liane ligneuse (énongué), le tout est bouilli pendant quelques minutes à une heure avec les lames des outils pour les empoisonner ; soit on utilise juste la poudre de l'écorce râpée, plus un peu d'eau, appliquée à la pointe des outils, puis emballée (par exemple avec une feuille de Marantaceae) et ficelée. On attendra au moins deux jours avant 
l'utilisation. D'après les dires de ces autochtones, le mélange de ces trois plantes rendrait encore le poison plus efficace et plus rapide que celui d'une seule espèce.

Nom scientifique : Strychnos camptoneura Gilg \& Busse

Nom vernaculaire : Énongué (aka)

C'est une grande liane grimpante. La plante est riche en alcaloïdes. L'écorce du tronc est utilisée en mélange avec celles de $S$. aculeata et d'ékombé (Apocynaceae) comme poison de flèche.

\section{*MENISPERMACEAE}

Nom scientifique : Penianthus longifolius Miers.

Nom vernaculaire : Ovoung grandes feuilles (français) ; Simbolo (aka)

Penianthus longifolius est un sousarbrisseau qui contient des alcaloïdes, d'où son usage comme ingrédient des poisons de flèche. Les fruits, pilés ensemble avec les feuilles et tiges de Periploca nigrescens, plus un scolopendre ou la glande à venin d'un serpent venimeux, donneraient un puissant poison de flèche dont on enduit tôt le matin les pointes des outils tout en demandant la bénédiction des mânes (les aïeux) et des "esprits grands chasseurs". Les jeunes tiges de $P$. longifolius servent de hampes pour les flèches.

\section{MORACEAE}

Nom scientifique : Ficus sp.

Nom vernaculaire : Ndémbo / Lémbo (aka)

C'est un grand arbre. On ne dispose pas d'information concernant ses propriétés biochimiques; mais les Aka Babènzè de la zone prospectée disent l'avoir utilisé pour empoisonner les pointes d'outils de chasse et l'exsudat bouilli dans un peu d'eau devient une pâte adhésive qu'on utilise pour piéger les oiseaux.

\section{PANDACEAE}

Nom scientifique : Panda oleosa Pierre.

Nom vernaculaire : Kanna (aka)

Arbre de petite taille, Panda oleosa ne contient pas d'alcaloïdes. On utilise ses fruits mûrs en voie de décomposition pour "déloger" le caïman de son trou, permettant ainsi sa capture ou sa mort; ils libèrent en effet une odeur plus ou moins forte qui serait insupportable pour l'animal.

\section{*PASSIFLORACEAE}

Nom scientifique : Adenia cissampeloides (Planch. ex Hook.) Harms.

Nom vernaculaire : Mõkõlõbõ (aka/ngbaka)

C'est une liane vigoureuse. La plante ne contient pas d'alcaloïdes mais des hétérosides cyanogénétiques (tétraphylline $\mathrm{B}$ et épitétraphylline), du gummiférol, un diépoxide polyacétylénique cytotoxique. Ses feuilles mélangées à celles de Periploca nigrescens sont pilées pour réduire en pâte qu'on applique aux pointes des outils. On jette dans une cour d'eau des tiges broyées pour anesthésier ou tuer les poissons.

\section{*PUTRANJIVACEAE}

Nom scientifique : Drypetes gossweileri $\mathrm{S}$. Moore.

Nom vernaculaire : Pandjo (aka)

Drypetes gossweileri est un arbre de taille moyenne. Les Aka de la Lobaye utilisent l'écorce râpée pour engourdir les poissons et l'écorce du tronc ou les feuilles pilées ensemble avec le tubercule de Dioscorea sp. donneraient un puissant poison de flèche. L'écorce du tronc se mange crue pour lutter contre les parasitoses.

\section{*RUBIACEAE}

Nom scientifique: indéterminé Nom vernaculaire: Molonzio (aka)

C'est un grand arbre. Les Aka utilisent ses écorces (écorcée ou râpée), plus ses feuilles ou ses fruits, réduits en pâte qu'ils appliquent aux tranchants des outils pour les empoisonner. Le poison reste actif pendant plus d'une semaine.

\section{*SAPOTACEAE}

Nom scientifique : Manilkara sp. (cf. M. multinervis (Bak.) Dubard.

Nom vernaculaire : Mò-ngénza (ngbaka/aka) 
Les Pygmées disent avoir utilisé l'écorce de ce petit arbre pour préparer un poison de flèche. Ses écorces mélangées à celles de Pentaclethra macrophylla, de litouma, de mokessa (Apocynacée) sont bouillies jusqu'à l'obtention d'un fluide sirupeux que l'on utilise pour enduire les pointes des outils.

Nom scientifique : indéterminé

Nom vernaculaire : Mboro / Mbolo (aka)

C'est un arbuste. L'échantillon a été prélevé au bord d'une piste en forêt secondaire. Les Aka utilisent les fruits avec les feuilles et tiges de Periploca nigrescens et le tubercule de Dioscorea sp., le tout pilé et réduit en pâte qu'on applique sur les tranchants d'outils.

Les espèces utilisées pour confectionner les arcs, les arbalètes, les sagaies

*EUPHORBIACEAE

Nom scientifique : Manniophyton fulvum Muell.Arg.

Nom vernaculaire : Mokossa (aka)

Manniophyton fulvum est une liane à tige cylindrique. Des traces d'alcaloïdes sont retrouvées dans ses feuilles. De cette plante, les Pygmées de la Lobaye tirent les cordes des arcs et arbalète, etc. et des ficelles pour le tissage des filets de chasse et de pêche. Elle n'est pas utilisée comme un poison ou comme un ingrédient.

\section{*URTICACEAE}

Nom scientifique : Myrianthus arboreus P. Beauv.

Nom vernaculaire : Ngoka (aka).

C'est un petit arbre. C'est grâce à cette plante que les Pygmées Aka fabriquent les manches de leur sagaie ou lance.

\section{*FAMILLES INDERMINÉES.}

Nom scientifique : indéterminé

Nom vernaculaire. Mokota (aka)

C'est un arbrisseau à exsudation de

latex rouge. L'espèce a été prélevée aux bords d'une piste en forêt secondaire. Les Aka utilisent les tiges pour confectionner les manches des sagaies.

Nom scientifique : indéterminé

Nom vernaculaire : Sètèkè (aka)

C'est une liane aux feuilles simples opposées. L'échantillon a été prélevé aux abords d'un village. La liane est utilisée pour fabriquer la corde des arcs, des arbalètes, etc.

\section{Les chaînes opératoires}

Les données ethnobotaniques accumulées grâce à ces enquêtes auprès des Pygmées Aka offrent une occasion de les éclairer par le formalisme posé à la base de l'ethnobotanique formelle (de Foucault, 1987). D'une manière très synthétique, il s'agit de décrire un fait botanique par la dualité structure/fonction: une plante initiale $\mathrm{P}$ (structure initiale) est soumise à une transformation (symbolisée par un opérateur formel du type a) par des hommes pour en faire un produit dérivé $\mathrm{P}^{\prime}$ (structure finale) pour un usage, une fonction $F^{\prime}$, de sorte que le fait ethnobotanique général peut s'écrire $(\mathbf{a P}=$ P', F'). Ce qui est notablement intéressant, c'est que les a sont relativement universels, étant indépendants des hommes qui les mettent en jeu et des plantes sur lesquelles ils jouent; ils peuvent même s'étendre à l'ethnozoologie, à l'ethnominéralogie... (de Foucault, 1992a, b). De manière plus complexe, plusieurs a peuvent se combiner en produit d'opérateurs élémentaires pour décrire la chaîne opératoire permettant de passer de $\mathrm{P}$ à $\mathrm{P}$ '.

Pour notre propos, nous mettrons en évidence quelques chaînes opératoires utilisant les opérateurs suivants :

1 : identité

e : emprunt d'une partie de $\mathrm{P}$ (écorce, feuilles, tige, fruits, tubercule...); la partie utilisée peut être précisée en indice de l'opérateur)

i : incision pour l'obtention de latex,...

p : préparation (par exemple ébullition d'un mélange) 
$\Sigma$ : structuration (à travers un mélange par exemple)

$\Delta$ : déstructuration (par écrasement...)

Par exemple Caladium bicolor $(\mathrm{Cb})$ est utilisé à travers la chaîne $\mathbf{p} \Delta \mathbf{e}_{\text {tubercule }} \mathrm{Cb}$ et Erythrophleum suaveolens (Es) par $\Delta \mathbf{e}_{\text {écorce }}$ Es ; Cascabela thevetia $(\mathrm{Ct})$ ou Elaeophorbia grandifolia (Eg) sont utilisés selon la simple chaîne $\mathbf{i}_{\text {latex }} T$ ou $\mathrm{Eg}$, ce qui nous permet d'écrire que Cascabela thevetia et Elaeophorbia grandifolia sont deux taxons homologues pour l'ethnobotanique des poisons végétaux car ils sont associés au même opérateur i pour la même fonction.

Deux plantes peuvent intervenir en mélange :

- Drypetes gossweileri (Dg) et Dioscorea sp. (D) par $\Sigma \Delta\left(\mathbf{e}_{\text {écorce ou }}\right.$ feuilles $\left.\mathrm{Dg}, \mathbf{e}_{\text {tubercule }} \mathrm{D}\right)$;

- Tabernaemontana crassa (Tc) et Periploca nigrescens (Pn) par $\Sigma \Delta\left(\mathbf{e}_{\text {feuilles }} \mathrm{Tc}, \mathbf{i}_{\text {latex }} \mathrm{Tc}, \mathbf{e}_{\text {feuilles }+ \text { tiges }} \mathrm{Pn}\right) ;$

- Mostuea hirsuta (Mh)et Periploca nigrescens (Pn) par $\Sigma \boldsymbol{\Delta}\left(\mathbf{e}_{\text {écorce }}\right.$ racinaire $\left.\mathrm{Mh}, \mathbf{e}_{\text {feuilles }} \mathrm{Pn}\right)$;

- Adenia cissampeloides (Ac) et Periploca nigrescens (Pn) par $\boldsymbol{\Sigma} \boldsymbol{\Delta}\left(\mathbf{e}_{\text {feuilles }} \mathrm{Ac}, \mathbf{e}_{\text {feuilles }} \mathrm{Pn}\right)$; ou trois :

- Strychnos aculeata (Sa), S. camptoneura $(\mathrm{Sc})$ et ékombé (é) par $\mathbf{p} \Delta\left(\mathbf{e}_{\text {écorce }} \mathrm{Sa}, \mathbf{e}_{\text {écorce }} \mathrm{Sc}, \mathbf{e}_{\text {écorce }}\right.$ ) ;

- Mboro (M), Dioscorea sp. (D) et Periploca nigrescens (Pn) par

$\boldsymbol{\Sigma} \boldsymbol{\Delta}\left(\mathbf{e}_{\text {fruits }} \mathrm{M}, \mathbf{e}_{\text {tubercule }} \mathrm{D}, \mathbf{e}_{\text {feuilles et tige }} \mathrm{Pn}\right) ;$ voire quatre: mokessa (m), Pentaclethra macrophylla (Pm), litouma (1), Manilkara sp. (M) par p $\boldsymbol{\Delta} \quad\left(\mathbf{e}_{\text {écorce }} \mathrm{m}, \mathbf{e}_{\text {écorce }} \mathrm{Pm}, \mathbf{e}_{\text {écorce }} \mathrm{l}\right.$, $\left.\mathbf{e}_{\text {écorce }} \mathbf{M}\right)$.

Plus rarement, le règne animal, avec des animaux toxiques ou à parties toxiques, peut accompagner le règne végétal pour une recette un peu plus complexe; c'est le cas avec Penianthus longifolius (Pl), Periploca nigrescens $(\mathrm{Pn})$, Scolopendre $(\mathrm{Sc})$ ou Serpent (Se) par la chaîne opératoire $\Sigma \Delta\left(\mathbf{e}_{\text {fruits }} \mathrm{Pl}, \mathbf{e}_{\text {feuilles }}\right.$ + tige $\mathrm{Pn}, \mathbf{1 S c}$ ou $\mathbf{e}_{\text {glande à venin }} \mathrm{Se}$ ). Dans ce dernier cas, l'intervention d'un rite (bénédiction des mânes et «esprits grands chasseurs » offre l'exemple d'un opérateur fonctionnel, et non plus structurel comme les a précédents (de Foucault \& Claisse, 1999): au-delà de la transformation structurelle en produit final, pour les Aka, la fonction F' ne sera vraiment remplie qu'après ce rite obligatoire.

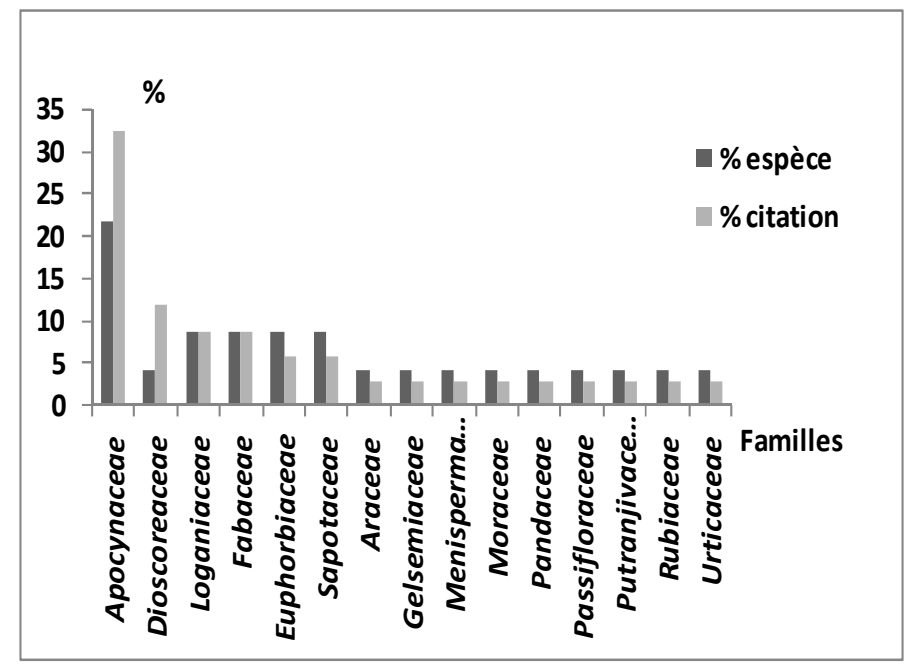

Figure 1 : Spectres brut (nombre d'espèce) et pondéré (nombre de citations) des familles. 


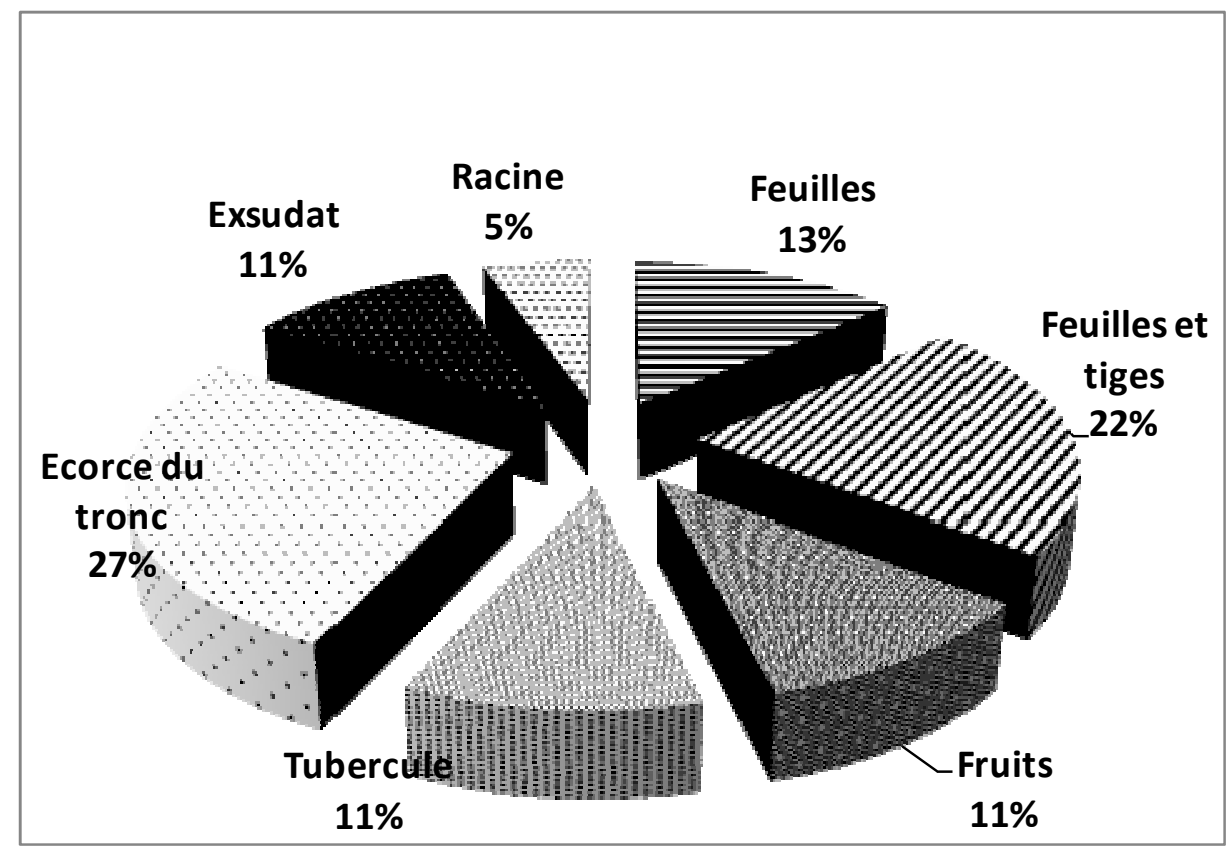

Figure 2 : Les organes végétaux utilisés dans les recettes.

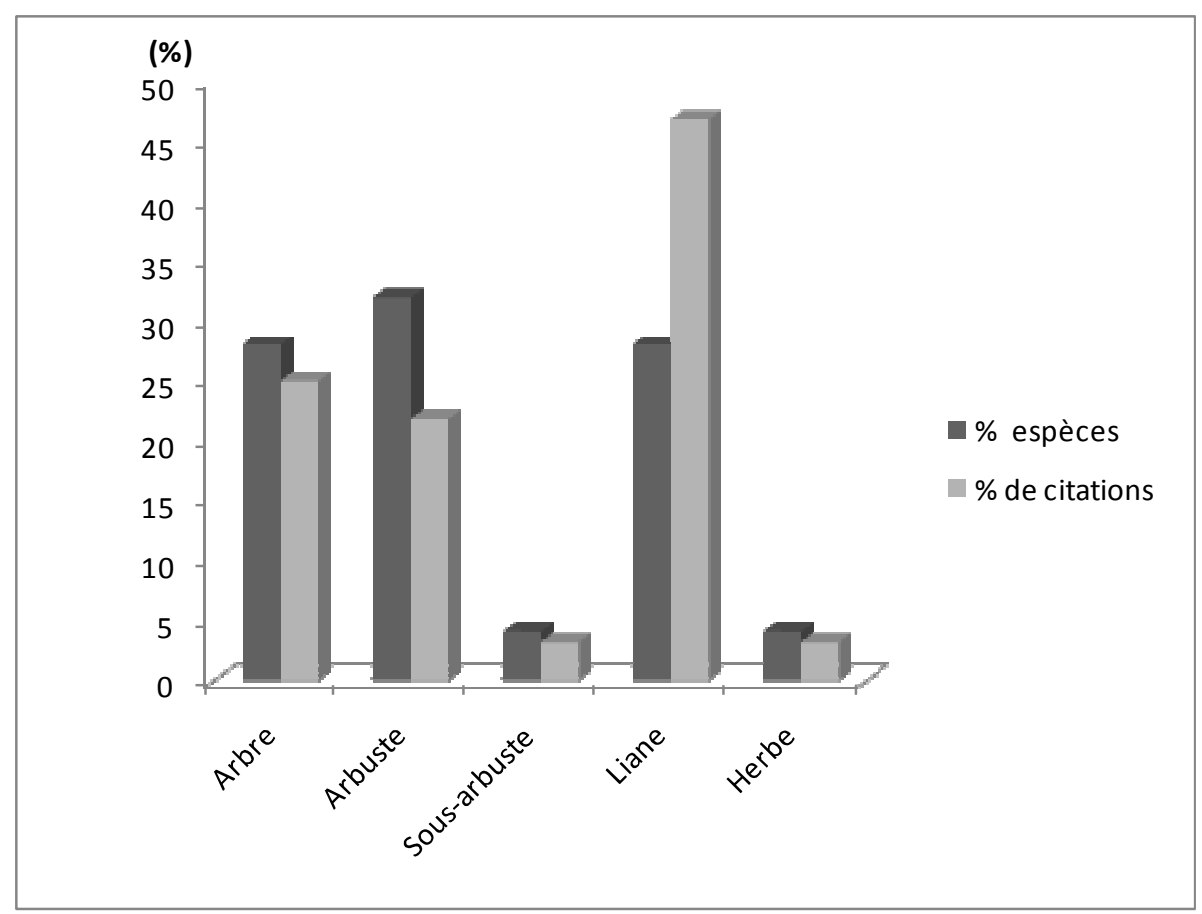

Figure 3 : Importance des différents types morphologiques des espèces inventoriées. 


\section{DISCUSSION}

L'utilisation des flèches empoisonnées est exclusivement masculine dans la région étudiée, ce qui confirme les résultats de Mathamal et al. (2009), qui précisent que, chez les Pygmées, la chasse avec les projectiles reste une activité réservée aux hommes. Ce sont surtout des adultes (40-60 ans) et des vieillards (plus de 60 ans) qui s'adonnent à ces pratiques. Ces valeurs ancestrales ne sont que de souvenirs dans cette contrée où on préfère les armes à feu et les pièges aux projectiles empoisonnés. Pour les jeunes générations, ce sont des pratiques révolues car disent-ils : «Ce sont des choses des Ko-tara (ancêtres) ; maintenant il y a des armes à feu, beaucoup plus indiquées pour la chasse de gros gibiers »; «Moi, je préfère bien les pièges (avec des câbles) et la cueillette du miel que cette pratique de mon grand-père, nécessitant quelquefois de parcourir de grandes distances ».

Pour cette étude, nous avons pu recenser 25 espèces végétales dont 21 sont exclusives à la préparation des poisons et les quatre autres utilisées accessoirement, avec deux indications concernant l'utilisation du matériel animal (serpents venimeux et scolopendres). Parmi les espèces déterminées, treize sont également signalées par d'autres auteurs : six par Vergiat (1970) (Periploca nigrescens, Penianthus longifolius, Dioscorea sp., les genres Strychnos et Euphorbia), cinq par Chevalier (1951) ( $P$. nigrescens, Dioscorea sp., Penianthus longifolius, Euphorbia sp. et le genre Erythrophleum), cinq par Raponda-Walker et Sillans (1961) ( $P$. nigrescens, Strychnos aculeata, Drypetes gossweileri, Manniophyton fulvum et le genre Erythrophleum), trois par Angenot (1978) (Periploca nigrescens, Dioscorea sp., le genre Erythrophleum et Penianthus longifolius), quatre par Motte-Florac (1980) (P. nigrescens, $S$. camptoneura et Euphorbia sp.), trois par Ruijter (2007, 2008a, b) (S. camptoneura, S. aculeata et Mostuea hirsuta), deux par Delaude (1978) ( $P$. nigrescens et $S$. camptoneura), deux par Schmelzer (2006) ( $S$. aculeata et Thevetia neriifolia), une par Grace et Powler (2007) (Adenia cissampeloides) et Mairura et Schmelzer (2006) (Tabernaemontana crassa), une par Newton (2008) (Elaeophorbia grandifolia).

En outre, comme Chevalier (1951), nous avons relevé que le venin des serpents venimeux entre dans la préparation de certains poisons de flèches en RCA. Comme l'a relevé Degroote (1960), nous constatons aussi que les poisons de base sont tirés de la famille des Apocynaceae. Il signale qu'en République Démocratique du Congo (RDC), ce sont les Strophantus kombe et $S$. gratus qui constituent les éléments de base de divers poisons alors qu'en Centrafrique, dans les localités étudiées, Periploca nigrescens est la plus citée. Elle est, en effet, beaucoup utilisée en association avec d'autres plantes. Nous pensons que cette espèce est incontestablement l'élément principal des poisons de flèche de la région de la Lobaye. Cette observation est conforme à celle de Motte-florac (1980) qui précise que $P$. nigrescens et Strychnos camptoneura constituent les plantes essentielles entrant dans la composition des poisons de flèches dans cette région. Terashima et al. (2008) relèvent aussi que $P$. nigrescens est le principal constituant du poison de flèche "mutali" en République Démocratique du Congo. Il faut reconnaître que certaines de ces espèces, comme Manilkara sp., Pentaclethra macrophylla, Myrianthus arboreus et Ficus sp., dépourvues d'alcaloïdes et d'hétérosides sont peut-être citées par erreur; ou alors elles jouent un rôle secondaire dans ces poisons (par exemple, la fixation du poison, le prolongement d'activité du poison,...). La toxicité de Caladium bicolor rend bien possible son utilisation dans cet art de l'empoisonnement, même si elle n'est connue que pour son usage ornemental, pharmacologique, etc.

\section{Conclusion}

En somme, on pourra retenir que cette étude nous a permis de relever 25 espèces végétales et deux citations de matériel animal 
(serpents venimeux et scolopendres), impliquant 22 recettes différentes de poisons de flèches. Periploca nigrescens constitue l'élément principal de ces poisons.

En outre, dans cette région de la Lobaye, chez les Pygmées, les pratiques de chasse à projectiles empoisonnés ne sont qu'un souvenir chez certaines personnes âgées. Leur mode de vie est profondément modifié. Ils ont tendance à s'orienter vers de nouvelles techniques de chasse et de pêche, et vers l'agriculture.

\section{REFERENCES}

Angenot L. 1978. Les poisons de flèches africains. 1. Zaïre, Rwanda et Burundi. Africa Tervuren, 24: 36-54.

Angiosperm Phylogeny Group. 2009. An update of the Angiosperm Phylogeny Group classification for the orders and families of flowering plants: APG III. Bot. J. Linn. Soc., 161: 105-121.

Aubréville A. 1948. Etude sur les forêts de l'Afrique Équatoriale Française et $d u$ Cameroun. Bull. Scient. $N^{\circ} 2$ : Nogent sur Marne.

Boulvert Y. 1996. Etude Géomorphologique de la République Centrafricaine. Carte à 1/1000000 en Deux Feuilles Ouest et Est. ORSTOM: Paris.

Chevalier A. 1951. Plantes-poisons de l'Oubangui et du moyen Congo. Revue Intern. Bot. Appl. Agric. Trop., 31(343344) : 249-257.

Ciccone B. 2011. Mécanisme d'action et pharmacologie des curares (http://brunociccone-pagesperso-orange.fr/). Consulté le 14.06. 2011.

Degroote V. 1960. Note Préliminaire sur les Flèches Empoisonnées et les Poisons de Flèches au Congo Belge. Service de Toxicologie de l'Institut de Médecine Tropicale : Léopoldville.

Delaude C. 1978. Les Végétaux du Zaïre: Matériel Médico-Magique des Guérisseurs et Sources de Recherche Phytogéographique. Centre de Coopération au Développement: Liège.
Flora of West Tropical Africa, 1958-1968. Vol. 1-3. C.A.O.G.A.: Millbank, London, S.W.1.

Flore du Cameroun. 1963-1991. 1-2, 4, 8-19, 24-25, 27-29, 32-33. Muséum National d'Histoire Naturelle, Paris ; M.E.S.I.R.S., Yaoundé.

Flore du Congo, du Rwanda et du Burundi, 1948-1968. Vol. 1-8 (1), 9-10, 12. INEAC, Jardin botanique nationale de Belgique : Bruxelles.

Flore du Gabon, 1961-1966. Tomes 1-29. Muséum National d'Histoire naturelle: Paris.

Foucault B (de). 1987. Essai de formalisation de l'ethnobotanique. J. Agric. Trad. Bot. Appl., 34: 31-45.

Foucault B (de). 1992a. Le domaine de l'ethnobotanique et ses extensions. Bull. Soc. Bot. N. France, 45: 57-64.

Foucault B (de). 1992b. De l'ethnobotanique à l'ethnoscience naturelle. Bull. Soc. Linn. N.-Picardie, 10: 15-34.

Foucault B (de), Claisse R. 1999. Le rite en tant qu'opérateur fonctionnel de l'ethnobotanique. In Incontrita medicine (Meetings between medicines), Guerci A (Éd), 124-132.

Grace OM, Powler D. 2007. Adenia cissampeloides (Plach. ex Hook.) Harms. Fiche de Protabase, Schmeizer GH, GuribFakim A (éds) PROTA (http://database.prota.org/)

Jassaud P. 1991. Histoire des hétérosides cardiotoniques. Actual. Pharmacol., 288: 69-75.

Kalk P. 1992. Histoire Centrafricaine. L'Harmattan: Paris.

Lebrun J-P, Stork AL. 1991. Enumération des Plantes à Fleurs d'Afrique Tropicale. Vol. 1. Généralités et Annonaceae à Pandaceae. Conservatoire et Jardin botaniques: Genève.

Lebrun J-P, Stork AL. 1992. Enumération des Plantes à Fleurs d'Afrique Tropicale. Vol. 2. Chrysobalanaceae à Apiaceae. Conservatoire et Jardin botaniques: Genève. 
Lebrun J-P, Stork AL. 1995. Enumération des Plantes à Fleurs d'Afrique Tropicale. Vol. 3. Monocotylédones: Limnocharitaceae à Poaceae. Conservatoire et Jardin botaniques: Genève.

Lebrun J-P, Stork AL. 1997. Enumération des Plantes à Fleurs d'Afrique Tropicale. Vol. 4. Gamopétales: Clethraceae à Lamiaceae. Conservatoire et Jardin botaniques : Genève.

Mairura FS, Schmelzer GH. 2006. Tabernaemontana crassa Benth. Fiche Protabase (http://database.prota.org/). Consulté le 1.09.2011.

Mathamal JJ, Sitamon St J, Mefp, Toumabia , Aidecor. 2009. Rapport sur la situation des peuples autochtones des forêts de la $R C A$. The Rain Forest Foundation: Bangui.

Motte-Florac E. 1980. Les plantes-poisons chez les Pygmées Aka et les Monzombo de la Lobaye. Thèse de doctorat $\left(3^{\mathrm{e}}\right.$ cycle $)$, Université de Paris, Paris, p. 573.

Newton LE. 2008. Elaeophorbia grandifolia (Haw.) Croizat. [Internet] Fiche de Protabase. Schmelzer GH, Gurib-Fakim A. (eds). PROTA (Plant Resources of Tropical Africa / Ressources végétales de l'Afrique tropicale): Wageningen, Pays Bas. (http://database.prota.org/recherche. htm). Consulté le 14.12.2011.
Raponda-Walker A, Sillans R. 1961. Les Plantes Utiles du Gabon. Lechevalier: Paris.

Ruijter A. 2007. Mostuea hirsuta. Fiche de Protabase (http://database.prota.org/). Consulté le 5.06.2011.

Ruijter A. 2008a. Strychnos camptoneura. Fiche de Protabase (http://database.prota. org/). Consulté le 5.06.2011.

Ruijter A. 2008b. Strychnos aculeata. Fiche de Protabase. (http://database.prota.org/). Consulté le 5.06.2011.

Schmelzer GH. 2006. Thevetia peruviana (Pers.) K.Schum. Fiche de Protabase. Schmelzer GH, Gurib-Fakim A. (Editeurs). PROTA (Plant Resources of Tropical Africa / Ressources végétales de l'Afrique tropicale), Wageningen, Pays Bas. (http://database.prota.org/recherche. htm). Consulté le 14.12.2011.

Terashima H, Ichikawa, Sawada M. 2008. Parquetina nigrescens (Afzel) Bullock. (http://WWW.ethnopharmacologie.rrg/). Consulté le 14.12.2011.

Vergiat AM. 1970. Plantes Magiques et Médicinales des Féticheurs de l'Oubangui. Laboratoire d'Ethnobotanique du Muséum National d'Histoire Naturelle: Paris. 